Provided for non-commercial research and education use. Not for reproduction, distribution or commercial use.

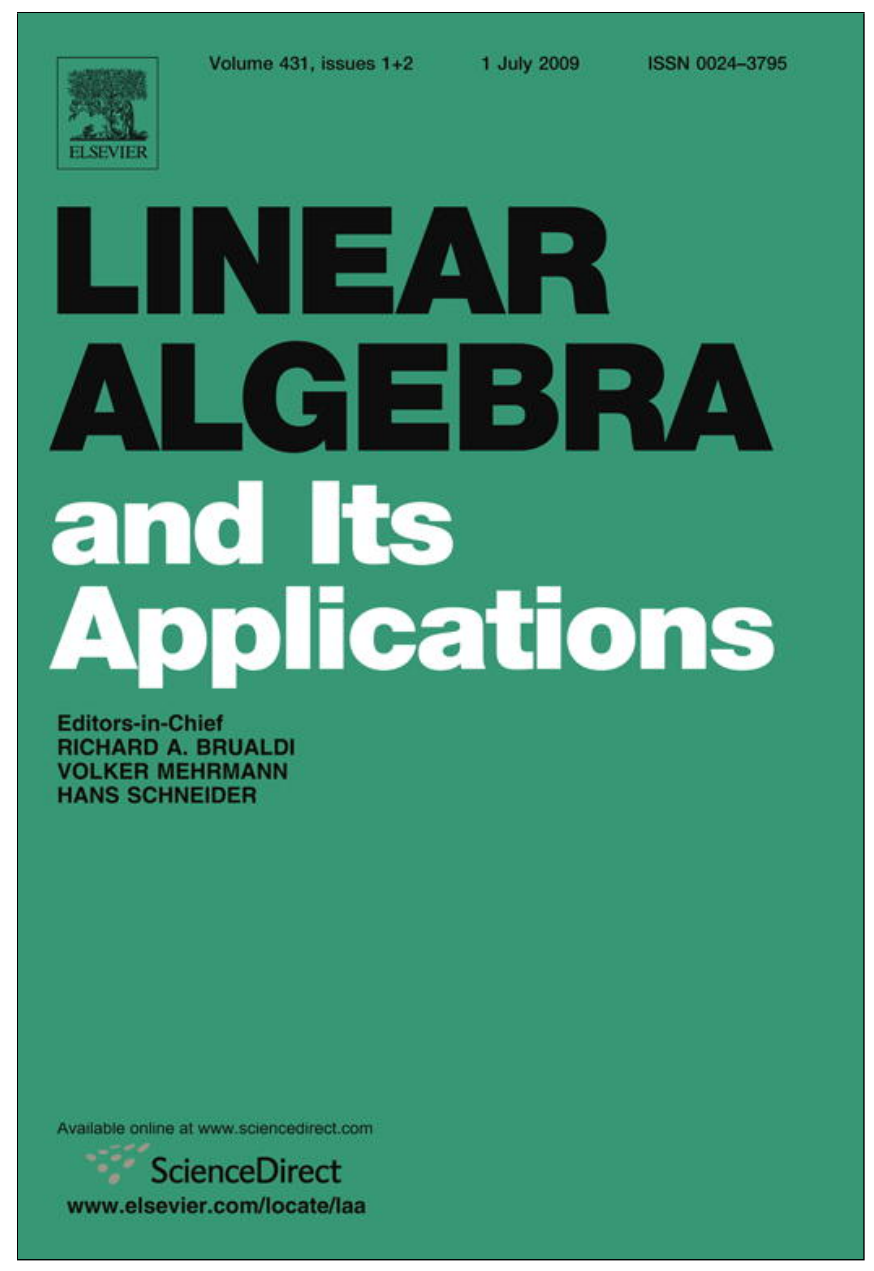

This article appeared in a journal published by Elsevier. The attached copy is furnished to the author for internal non-commercial research and education use, including for instruction at the authors institution and sharing with colleagues.

Other uses, including reproduction and distribution, or selling or licensing copies, or posting to personal, institutional or third party websites are prohibited.

In most cases authors are permitted to post their version of the article (e.g. in Word or Tex form) to their personal website or institutional repository. Authors requiring further information regarding Elsevier's archiving and manuscript policies are encouraged to visit:

http://www.elsevier.com/copyright 


\title{
Strict diagonal dominance and a Geršgorin type theorem in Euclidean Jordan algebras
}

\author{
Melania M. Moldovan *, M. Seetharama Gowda \\ Department of Mathematics and Statistics, University of Maryland, Baltimore County, Baltimore, \\ MD 21250, United States
}

\section{A R T I C L E I N F O}

\section{Article history:}

Received 14 November 2008

Accepted 15 February 2009

Available online 25 March 2009

Submitted by H. Schneider

\section{Keywords:}

Quaternions

Octonions

Euclidean Jordan algebras

Strict diagonal dominance

Geršgorin type theorem

\begin{abstract}
A B S T R A C T
For complex square matrices, the Levy-Desplanques theorem asserts that a strictly diagonally dominant matrix is invertible. The well-known Geršgorin theorem on the location of eigenvalues is equivalent to this. In this article, we extend the Levy-Desplanques theorem to an object in a Euclidean Jordan algebra when its Peirce decomposition with respect to a Jordan frame is given. As a consequence, we prove a Geršgorin type theorem for the spectral eigenvalues of an object in a Euclidean Jordan algebra.

(C) 2009 Elsevier Inc. All rights reserved.
\end{abstract}

\section{Introduction}

In matrix theory, the well-known Geršgorin theorem [10] asserts that for an $n \times n$ complex matrix $A=\left[a_{i j}\right]$, the spectrum (consisting of the eigenvalues) of $A$ lies in the union of Geršgorin discs in the complex plane:

$$
\sigma(A) \subseteq \bigcup_{i=1}^{n}\left\{z \in C:\left|z-a_{i i}\right| \leqslant R_{i}(A)\right\}
$$

where

$$
R_{i}(A):=\sum_{j=1, j \neq i}^{n}\left|a_{i j}\right| \quad(1 \leqslant i \leqslant n) .
$$

* Corresponding author.

E-mail addresses: melania1@math.umbc.edu (M.M. Moldovan), gowda@math.umbc.edu (M.S. Gowda). 
This is equivalent to the strict diagonal dominance theorem - known as the Levy-Desplanques theorem [10] - which says that if an $n \times n$ complex matrix $A=\left[a_{i j}\right]$ is strictly diagonally dominant, that is,

$$
\left|a_{i i}\right|>R_{i}(A) \quad \forall i=1,2, \ldots, n,
$$

then $A$ is invertible in $C^{n \times n}$.

In a recent paper [15], Zhang extends the Geršgorin theorem to quaternionic matrices by stating two results, one for left eigenvalues and the other one for right eigenvalues (the difference arising because of non-commutative nature of quaternions). The strict diagonal dominance result extends to quaternionic matrices, since for a quaternionic square matrix $A$, the following two conditions are equivalent [14]:

(a) $A x=0 \Rightarrow x=0$.

(b) $A$ is invertible, that is, there is a quaternionic matrix $B$ such that $A B=B A=I$.

It is easily seen (see Section 4) that Zhang's two Geršgorin type results carry over to octonionic matrices. Furthermore, the strict diagonal dominance condition implies condition (a) above and a modified version of (b).

Our objective in this paper is to prove analogs of the above results in Euclidean Jordan algebras. More precisely, we show that if $(V, \circ,\langle\cdot, \cdot\rangle)$ is a Euclidean Jordan algebra of rank $r$ and

$$
x=\sum_{i=1}^{r} x_{i} e_{i}+\sum_{i<j} x_{i j}
$$

is the Peirce decomposition of $x \in V$ with respect to a given Jordan frame $\left\{e_{1}, \ldots, e_{r}\right\}$ (see Section 3 for definitions), then the strict diagonal dominance condition

$$
\left|x_{i}\right|>R_{i}(x):=\frac{1}{\sqrt{2}\left\|e_{i}\right\|}\left(\sum_{k=1}^{i-1}\left\|x_{k i}\right\|+\sum_{j=i+1}^{r}\left\|x_{i j}\right\|\right) \quad \forall i=1,2, \ldots, r
$$

implies the invertibility of $x$ in $V$. Moreover, for any $x \in V$, we have

$$
\sigma_{s p}(x) \subseteq \bigcup_{i=1}^{r}\left\{\lambda \in R:\left|\lambda-x_{i}\right| \leqslant R_{i}(x)\right\},
$$

where $\sigma_{s p}(x)$ denotes the set of all spectral eigenvalues (coming from the spectral decomposition) of $x$ in $V$. As a consequence, we deduce that if each $x_{i}$ is positive and the strict diagonal dominance condition holds, then $x$ is in the interior of the symmetric cone in $V$.

Our analysis is as follows. Since the results for real/complex Hermitian matrices are known, we first prove the strict diagonal dominance result in the matrix algebras of $n \times n$ quaternion Hermitian matrices, $3 \times 3$ octonion Hermitian matrices, and the Jordan spin algebra. Then we use the structure theorem - that any Euclidean Jordan algebra is essentially the product of above mentioned algebras to cover the general case. From this, we easily deduce the Geršgorin type result mentioned above. As we shall see, the case of $3 \times 3$ octonion Hermitian matrices requires special consideration: for such matrices, the spectral eigenvalues can be different from the real left/right eigenvalues and the strict diagonal dominance result requires a non-standard proof that avoids left/right eigenvalues.

Our paper is organized as follows. In Section 2, we describe matrices over quaternions and octonions. In Section 3, we cover Euclidean Jordan algebra concepts, examples, and all preliminary results. In Section 4, we describe Geršgorin type results for left/right eigenvalues of matrices with entries from real numbers/complex numbers/quaternions/octonions. Section 5 covers the strict diagonal dominance results for matrices. In Section 6, we prove the strict diagonal dominance result in Euclidean Jordan algebras. Finally, in Section 7, we prove a Geršgorin type theorem in Euclidean Jordan algebras.

\section{Square matrices over quaternions and octonions}

Throughout this paper, we use the standard notations $-R$ for the set of all real numbers and $C$ for the set of all complex numbers. 
The linear space of quaternions - denoted by $\mathcal{H}$ - is a 4-dimensional linear space over $R$ with a basis $\{1, i, j, k\}$. The space $\mathcal{H}$ is made into an algebra by means of the conditions

$$
i^{2}=j^{2}=k^{2}=-1 \text { and } i j k=-1 \text {. }
$$

For any $x=x_{0} 1+x_{1} i+x_{2} j+x_{3} k \in \mathcal{H}$, we define the real part and conjugate by

$$
\operatorname{Re}(x):=x_{0} \text { and } \bar{x}:=x_{0} 1-x_{1} i-x_{2} j-x_{3} k .
$$

The linear space of octonions over $R$ - denoted by $\mathcal{O}$ - is an 8-dimensional linear space with basis $\left\{1, e_{1}, e_{2}, e_{3}, e_{4}, e_{5}, e_{6}, e_{7}\right\}$. The space $\mathcal{O}$ becomes an algebra via the following multiplication table on the non-unit basis elements [13]:

\begin{tabular}{rrrrrrrr}
\hline & $e_{1}$ & $e_{2}$ & $e_{3}$ & $e_{4}$ & $e_{5}$ & $e_{6}$ & $e_{7}$ \\
\hline$e_{1}$ & -1 & $e_{3}$ & $-e_{2}$ & $e_{5}$ & $-e_{4}$ & $-e_{7}$ & $-e_{6}$ \\
$e_{2}$ & $-e_{3}$ & -1 & $e_{1}$ & $e_{6}$ & $e_{7}$ & $-e_{4}$ & $-e_{5}$ \\
$e_{3}$ & $e_{2}$ & $-e_{1}$ & -1 & $e_{7}$ & $-e_{6}$ & $e_{5}$ & $-e_{4}$ \\
$e_{4}$ & $-e_{5}$ & $-e_{6}$ & $-e_{7}$ & -1 & $e_{1}$ & $e_{2}$ & $e_{3}$ \\
$e_{5}$ & $e_{4}$ & $-e_{7}$ & $e_{6}$ & $-e_{1}$ & -1 & $-e_{3}$ & $e_{2}$ \\
$e_{6}$ & $e_{7}$ & $e_{4}$ & $-e_{5}$ & $-e_{2}$ & $e_{3}$ & -1 & $-e_{1}$ \\
$e_{7}$ & $-e_{6}$ & $e_{5}$ & $e_{4}$ & $-e_{3}$ & $-e_{2}$ & $e_{1}$ & -1 \\
\hline
\end{tabular}

For an element

$$
x=x_{0} 1+x_{1} e_{1}+x_{2} e_{2}+x_{3} e_{3}+x_{4} e_{4}+x_{5} e_{5}+x_{6} e_{6}+x_{7} e_{7}
$$

in $\mathcal{O}$, we define the real part and conjugate by $\operatorname{Re}(x):=x_{0}$ and

$$
\bar{x}=x_{0}-x_{1} e_{1}-x_{2} e_{2}-x_{3} e_{3}-x_{4} e_{4}-x_{5} e_{5}-x_{6} e_{6}-x_{7} e_{7} .
$$

In both $\mathcal{H}$ and $\mathcal{O}$, we define the norm by $|x|=\sqrt{x \bar{x}}$. In these spaces, $|x y|=|x||y|$ for all $x$ and $y$. It is well known that $\mathcal{H}$ and $\mathcal{O}$ are non-commutative normed division algebras, and while $\mathcal{H}$ is associative, $\mathcal{O}$ is not.

Let $F$ denote the set of all reals/complex numbers/quaternions/octonions. We write $F^{n}$ for the space of all $n \times 1$ vectors over $F$ and $F^{n \times n}$ for the space of all $n \times n$ matrices over $F$. For a matrix $A \in F^{n \times n}$, we define the conjugate $\bar{A}$ and transpose $A^{T}$ in the usual way. We say that a square matrix $A \in F^{n \times n}$ is Hermitian if $A$ coincides with its conjugate transpose, that is, if $A=A^{*}:=(\bar{A})^{T}$. We let

$\operatorname{Herm}\left(F^{n \times n}\right):=$ set of all $\mathrm{n} \times \mathrm{n}$ Hermitian matrices with entries from $F$.

For a matrix $A \in F^{n \times n}$, an element $\lambda \in F$ is a left (right) eigenvalue of $A$ if there is a nonzero $x \in F^{n}$ such that $A x=\lambda x$ (respectively, $A x=x \lambda$ ). We use the notation $\sigma_{l}(A)\left(\sigma_{r}(A)\right)$ for the set of all left eigenvalues of $A$ (respectively, the right eigenvalues of $A$ ).

For discussions on eigenvalues of quaternionic/octonionic matrices, we refer to $[2-5,14,15]$.

Theorem 1. The following statements hold:

(a) Let $A \in \mathcal{H}^{n \times n}$. The implication $\left[x \in \mathcal{H}^{n}, A x=0\right] \Rightarrow x=0$ holds if and only if there is a unique $B \in \mathcal{H}^{n \times n}$ such that $A B=B A=I[14$, Theorem 4.3].

(b) Let $A \in \operatorname{Herm}\left(\mathcal{H}^{n \times n}\right)$. Then there exist real eigenvalues $\lambda_{1}, \lambda_{2}, \ldots, \lambda_{n}$ and corresponding eigenvectors $v_{1}, v_{2}, \ldots, v_{n}$ in $\mathcal{H}^{n}$ such that

$v_{i}^{*} v_{j}=\delta_{i j} \quad(\forall i, j)$,

$A=\sum_{m=1}^{n} \lambda_{m} v_{m} v_{m}^{*}$ and $I=\sum_{m=1}^{n} v_{m} v_{m}^{*}$

(Theorem $\left.1_{\mathcal{H}},[3]\right)$. 
(c) Let $A \in \mathcal{O}^{n \times n}$. The implication $\left[x \in \mathcal{O}^{n}, A x=0\right] \Rightarrow x=0$ holds if and only if there exist unique $B$ and $C$ in $\mathcal{O}^{n \times n}$ such that $A B=C A=I[13$, Lemma 4.4, Theorem 4.13, and Corollary 4.14].

\section{Euclidean Jordan algebras}

In this section, we briefly recall concepts, properties/results, and examples from Euclidean Jordan algebra theory. For short introductions, see [8,11]. For complete details, we refer to [6].

A Euclidean Jordan algebra [6] is a triple $(V, \circ,\langle.,\rangle$.$) , where (V,\langle\cdot, \cdot\rangle)$ is a finite-dimensional inner product space over $R$ and $(x, y) \mapsto x \circ y: V \times V \rightarrow V$ is a bilinear mapping satisfying the following conditions for all $x, y$, and $z: x \circ y=y \circ x, x \circ\left(x^{2} \circ y\right)=x^{2} \circ(x \circ y)$, and $\langle x \circ y, z\rangle=\langle y, x \circ z\rangle$. In addition, we assume that there is an element $e \in V$ (called the unit element) such that $x \circ e=x$, for all $x \in V$. The so-called symmetric cone of $V$ is given by $K:=\{x \circ x: x \in V\}$. This is a closed convex self-dual cone.

A Euclidean Jordan algebra is said to be simple if it is non-trivial and it cannot be written as the product of two (non-trivial) Euclidean Jordan algebras.

We now state the structure theorem for Euclidean Jordan algebras. In the matrix algebras below, we define the Jordan and inner product by:

$$
X \circ Y:=\frac{1}{2}(X Y+Y X) \text { and }\langle X, Y\rangle:=\operatorname{Re} \operatorname{trace}(X Y),
$$

where the trace (abbreviated as 'tr') of a matrix is the sum of its diagonal entries.

Theorem 2 (The structure theorem for Euclidean Jordan algebras [6]). Every non-trivial Euclidean Jordan algebra is a product of simple Euclidean Jordan algebras and every simple algebra is isomorphic to one of the following:

(i) The space Herm $\left(R^{n \times n}\right)$ of all $n \times n$ real symmetric matrices.

(ii) The space Herm $\left(C^{n \times n}\right)$ of all $n \times n$ complex Hermitian matrices.

(iii) The space Herm $\left(\mathcal{H}^{n \times n}\right)$ of all $n \times n$ quaternionic Hermitian matrices.

(iv) The space Herm $\left(\mathcal{O}^{3 \times 3}\right)$ of all $3 \times 3$ octonionic Hermitian matrices.

(v) The Jordan spin algebra $\mathcal{L}^{n}$ for $n>1$.

(See Example 2 below for a description of $\mathcal{L}^{n}$.)

An element $c \in V$ is an idempotent if $c^{2}=c$; it is a primitive idempotent if it is nonzero and cannot be written as a sum of two nonzero idempotents. We say that a finite set $\left\{e_{1}, e_{2}, \ldots, e_{r}\right\}$ of primitive idempotents in $V$ is a Jordan frame if

$$
e_{i} \circ e_{j}=0 \text { if } i \neq j \text { and } e_{1}+e_{2}+\cdots+e_{r}=e .
$$

For $x \in V$, we define $m(x):=\min \left\{k>0: e, x, \ldots, x^{k}\right.$ are linearly dependent $\}$ and rank of $V$ by $r=$ $\max \{m(x): x \in V\}$.

Theorem 3 (Spectral decomposition theorem). Let $V$ be a Euclidean Jordan algebra with rank $r$. Then for every $x \in V$, there exist a Jordan frame $\left\{e_{1}, e_{2}, \ldots, e_{r}\right\}$ and real numbers $\lambda_{1}, \ldots, \lambda_{r}$ such that

$$
x=\lambda_{1} e_{1}+\cdots+\lambda_{r} e_{r} .
$$

The numbers $\lambda_{i}$ are called the spectral eigenvalues of $x$. (In this paper, we have used the additional word 'spectral' in order to distinguish these eigenvalues from the left/right eigenvalues of matrices.)

Given the spectral eigenvalues of $x$, we define

$$
\begin{aligned}
& \sigma_{s p}(x):=\left\{\lambda_{1}, \lambda_{2}, \ldots, \lambda_{r}\right\}, \\
& \operatorname{trace}(x):=\lambda_{1}+\lambda_{2}+\cdots+\lambda_{r}, \text { and } \operatorname{det}(x):=\lambda_{1} \lambda_{2} \cdots \lambda_{r} .
\end{aligned}
$$


Corresponding to an $x \in V$, we define the Lyapunov operator $L_{x}$ on $V$ by $L_{x}(z):=x \circ z$. We say that two elements $x$ and $y$ in $V$ operator commute if the corresponding Lyapunov operators $L_{x}$ and $L_{y}$ commute (which can happen if and only if $x$ and $y$ have their spectral decompositions with respect to the same Jordan frame [6]).

We say that an element $x$ is invertible in $V$ if all the spectral eigenvalues of $x$ are nonzero. This happens if and only if there is a $y$ in $V$ that operator commutes with $x$ and $x \circ y=e$.

Given a Euclidean Jordan algebra $V$, an invertible linear transformation $\Lambda: V \rightarrow V$ is said to be an algebra automorphism if

$$
\Lambda(x \circ y)=\Lambda(x) \circ \Lambda(y) \quad \forall x, y \in V .
$$

We need the following results for our later use:

- The trace and determinant are invariant under algebra automorphisms.

- In a simple Euclidean Jordan algebra, every algebra automorphism is orthogonal (that is, it preserves the inner product), see p. 56 [6].

- In a simple algebra, any Jordan frame can be mapped onto any other Jordan frame by an algebra automorphism, see Theorem IV.2.5 [6].

Let $\left\{e_{1}, e_{2}, \ldots, e_{r}\right\}$ be a Jordan frame in a Euclidean Jordan algebra $V$. For $i, j \in\{1,2, \ldots, r\}$, we define the Peirce eigenspaces

$$
V_{i i}:=\left\{x \in V: x \circ e_{i}=x\right\}=R e_{i}
$$

and when $i \neq j$,

$$
V_{i j}:=\left\{x \in V: x \circ e_{i}=\frac{1}{2} x=x \circ e_{j}\right\} .
$$

Theorem 4 (Theorem IV.2.1, [6]). The space $V$ is the orthogonal direct sum of spaces $V_{i j}(i \leqslant j)$.

Thus, given a Jordan frame $\left\{e_{1}, e_{2}, \ldots, e_{r}\right\}$, we can write any element $x \in V$ as

$$
x=\sum_{i=1}^{r} x_{i} e_{i}+\sum_{i<j} x_{i j}
$$

where $x_{i} \in R$ and $x_{i j} \in V_{i j}$. This expression is the Peirce decomposition of $x$ with respect to $\left\{e_{1}, e_{2}, \ldots, e_{r}\right\}$. Given the above Peirce decomposition of $x$, we define the Geršgorin radii of $x$ :

$$
R_{i}(x):=\frac{1}{\sqrt{2}\left\|e_{i}\right\|}\left(\sum_{k=1}^{i-1}\left\|x_{k i}\right\|+\sum_{j=i+1}^{r}\left\|x_{i j}\right\|\right), \quad i=1,2, \ldots, r .
$$

We illustrate this in the following examples.

Example 1. In the matrix algebras $\operatorname{Herm}\left(F^{n \times n}\right)$ (with $n=3$ when $F=\mathcal{O}$ ), the set $\left\{E_{1}, E_{2}\right.$, $\left.\ldots, E_{n}\right\}$ is a Jordan frame, where $E_{i}$ is the diagonal matrix with 1 in the $(i, i)$-slot and zeros elsewhere. For a matrix $X$ in any one of these algebras, it is easy to write down the Peirce decomposition with respect to $\left\{E_{1}, E_{2}, \ldots, E_{n}\right\}$ and compute the Geršgorin radii. For example, in $\operatorname{Herm}\left(\mathcal{O}^{3 \times 3}\right)$,

$$
X=\left[\begin{array}{ccc}
p & a & b \\
\bar{a} & q & c \\
\bar{b} & \bar{c} & r
\end{array}\right]=p E_{1}+q E_{2}+r E_{3}+X_{12}+X_{13}+X_{23},
$$

where

$$
X_{12}=\left[\begin{array}{lll}
0 & a & 0 \\
\bar{a} & 0 & 0 \\
0 & 0 & 0
\end{array}\right], \quad X_{13}=\left[\begin{array}{ccc}
0 & 0 & b \\
0 & 0 & 0 \\
\bar{b} & 0 & 0
\end{array}\right], \quad \text { and } X_{23}=\left[\begin{array}{ccc}
0 & 0 & 0 \\
0 & 0 & c \\
0 & \bar{c} & 0
\end{array}\right] \text {. }
$$


Corresponding to this, we have (the Geršgorin radii of $X$ ):

$$
\begin{aligned}
& R_{1}(X)=\frac{1}{\sqrt{2}\left\|E_{1}\right\|}\left(\left\|X_{12}\right\|+\left\|X_{13}\right\|\right)=|a|+|b|, \\
& R_{2}(X)=\frac{1}{\sqrt{2}\left\|E_{2}\right\|}\left(\left\|X_{12}\right\|+\left\|X_{23}\right\|\right)=|a|+|c|,
\end{aligned}
$$

etc.

More generally, for an object $A=\left[a_{i j}\right] \in \operatorname{Herm}\left(F^{n \times n}\right)$ (with $n=3$ when $F=\mathcal{O}$ ), it is easily seen that with respect to the Jordan frame $\left\{E_{1}, E_{2}, \ldots, E_{n}\right\}$,

$$
R_{i}(A):=\sum_{j=1, j \neq i}^{n}\left|a_{i j}\right| \quad(1 \leqslant i \leqslant n) .
$$

Example 2. Consider the Jordan spin algebra $\mathcal{L}^{n}$ whose underlying space is $R^{n}, n>1$. We write any element $x$ in the form

$$
x=\left[\begin{array}{c}
x_{0} \\
\bar{x}
\end{array}\right]
$$

with $x_{0} \in R$ and $\bar{x} \in R^{n-1}$. The inner product in $\mathcal{L}^{n}$ is the usual inner product on $R^{n}$. The Jordan product $x \circ y$ in $\mathcal{L}^{n}$ is defined by

$$
x \circ y=\left[\begin{array}{c}
x_{0} \\
\bar{x}
\end{array}\right] \circ\left[\begin{array}{c}
y_{0} \\
\bar{y}
\end{array}\right]:=\left[\begin{array}{c}
\langle x, y\rangle \\
x_{0} \bar{y}+y_{0} \bar{x}
\end{array}\right] .
$$

Then $\mathcal{L}^{n}$ is a Euclidean Jordan algebra of rank 2 and for any element $x \in \mathcal{L}^{n}$, see Example 10 in [11],

$$
\operatorname{det}(x)=x_{0}^{2}-\|\bar{x}\|^{2} \text {. }
$$

Now consider any Jordan frame $\left\{e_{1}, e_{2}\right\}$ in $\mathcal{L}^{n}$. Then there exists a unit vector $u \in R^{n-1}$ such that

$$
e_{1}:=\frac{1}{2}\left[\begin{array}{l}
1 \\
u
\end{array}\right] \text { and } e_{2}:=\frac{1}{2}\left[\begin{array}{c}
1 \\
-u
\end{array}\right] \text {. }
$$

With respect to this, any $x \in \mathcal{L}^{n}$ given by (4) has a Peirce decomposition

$$
\left[\begin{array}{c}
x_{0} \\
\bar{x}
\end{array}\right]=x_{1} e_{1}+x_{2} e_{2}+x_{12}=x_{1} \frac{1}{2}\left[\begin{array}{l}
1 \\
u
\end{array}\right]+x_{2} \frac{1}{2}\left[\begin{array}{c}
1 \\
-u
\end{array}\right]+\left[\begin{array}{l}
0 \\
v
\end{array}\right] \text {, }
$$

where $v \in R^{n-1}$ with $\langle u, v\rangle=0$. (This is easy to verify, see e.g., Lemma 2.3.4 [12].) This leads to

$$
x_{0}=\frac{1}{2}\left(x_{1}+x_{2}\right) \text { and } \bar{x}=\frac{1}{2}\left(x_{1}-x_{2}\right) u+v .
$$

Thus

$$
\operatorname{det}(x)=x_{0}^{2}-\|\bar{x}\|^{2}=x_{1} x_{2}-\|v\|^{2}=x_{1} x_{2}-\left\|x_{12}\right\|^{2} .
$$

We finally note that as $\left\|e_{1}\right\|=\left\|e_{2}\right\|=\frac{1}{\sqrt{2}}$, the Geršgorin radii of $x$ are given by

$$
R_{1}(x)=\frac{1}{\sqrt{2}\left\|e_{1}\right\|}\left\|x_{12}\right\|=\left\|x_{12}\right\|=R_{2}(x) .
$$

The algebra $\operatorname{Herm}\left(\mathcal{O}^{3 \times 3}\right)$ is crucial for our analysis. We collect below two important results that are needed.

For $A, B \in \operatorname{Herm}\left(\mathcal{O}^{3 \times 3}\right)$, the so-called Freudenthal product [3] is defined by

$$
A * B:=A \circ B-\frac{1}{2}(A \operatorname{tr}(B)+B \operatorname{tr}(A))+\frac{1}{2}(\operatorname{tr}(A) \operatorname{tr}(B)-\operatorname{tr}(A \circ B)) I,
$$


where $I$ is the identity matrix. Recall that for a matrix $A \in \operatorname{Herm}\left(\mathcal{O}^{3 \times 3}\right)$, $\operatorname{det}(A)$ is the product of its spectral eigenvalues. In the result below (which is essentially in [3]), we express this determinant in terms of the entries of $A$.

Lemma 5. Let $A \in \operatorname{Herm}\left(\mathcal{O}^{3 \times 3}\right)$ be given by

$$
A:=\left[\begin{array}{lll}
p & a & b \\
\bar{a} & q & c \\
\bar{b} & \bar{c} & r
\end{array}\right],
$$

where $p, q, r \in R$ and $a, b, c \in \mathcal{O}$. Then

$$
\operatorname{det}(A)=\frac{1}{3} \operatorname{tr}((A * A) \circ A)=p q r+2 \operatorname{Re}(\bar{b}(a c))-r|a|^{2}-q|b|^{2}-p|c|^{2} .
$$

Proof. The second equality comes from direct computation, see [3]. In particular, when $A$ is diagonal, the middle expression reduces to the product of the diagonal entries of $A$.

We prove the first equality. By the spectral decomposition theorem, we may write $A=\lambda_{1} f_{1}+$ $\lambda_{2} f_{2}+\lambda_{3} f_{3}$, where $\lambda_{1}, \lambda_{2}, \lambda_{3}$ are the spectral eigenvalues of $A$, and $\left\{f_{1}, f_{2}, f_{3}\right\}$ is a Jordan frame in $\operatorname{Herm}\left(\mathcal{O}^{3 \times 3}\right)$. As this algebra is simple, there is an algebra automorphism $\Lambda$ of $\operatorname{Herm}\left(\mathcal{O}^{3 \times 3}\right)$ that maps $\left\{f_{1}, f_{2}, f_{3}\right\}$ to $\left\{E_{1}, E_{2}, E_{3}\right\}$, where $E_{i}$ is a $3 \times 3$ matrix with one in the $(i, i)$ slot and zeros elsewhere. Then $\Lambda(A)$ is a diagonal matrix with $\lambda_{1}, \lambda_{2}, \lambda_{3}$ on the diagonal. Since

$$
\Lambda(A \circ B)=\Lambda(A) \circ \Lambda(B), \quad \Lambda(A * B)=\Lambda(A) * \Lambda(B) \text { and } \operatorname{tr} \Lambda(A)=\operatorname{tr}(A),
$$

we have (from the second equality in (7) applied to $\Lambda(A)$ ),

$$
\frac{1}{3} \operatorname{tr}((\Lambda(A) * \Lambda(A)) \circ \Lambda(A))=\lambda_{1} \lambda_{2} \lambda_{3} .
$$

But

$$
\frac{1}{3} \operatorname{tr}((\Lambda(A) * \Lambda(A)) \circ \Lambda(A))=\frac{1}{3} \operatorname{tr} \Lambda((A * A) \circ A)=\frac{1}{3} \operatorname{tr}((A * A) \circ A) .
$$

Thus,

$$
\operatorname{det}(A)=\lambda_{1} \lambda_{2} \lambda_{3}=\frac{1}{3} \operatorname{tr}((A * A) \circ A)
$$

proving the first equality in (7).

For objects $a, b, c \in \mathcal{O}$ and for the matrix $A$ given above, we let

$$
[a, b]:=a b-b a, \quad[a, b, c]:=(a b) c-a(b c)
$$

and $\Phi(a, b, c):=\frac{1}{2} \operatorname{Re}([a, b] c)$. Also, let

$$
s(A):=p q+q r+r p-|a|^{2}-|b|^{2}-|c|^{2} .
$$

(Recall that $\operatorname{tr}(A)=p+q+r$.)

Remark 1. It follows from (7) that the spectral eigenvalues of the above $A$ are the roots of

$$
\operatorname{det}(\lambda I-A)=\lambda^{3}-(\operatorname{tr} A) \lambda^{2}+s(A) \lambda-\operatorname{det}(A)=0 .
$$

We need the following result from [3] which was verified using Mathematica.

Lemma 6 (Lemma $1_{\mathrm{O}_{3}}$, [3]). The real eigenvalues of the $3 \times 3$ octonion Hermitian matrix A satisfy the modified characteristic equation

$$
\operatorname{det}(\lambda I-A)=\lambda^{3}-(\operatorname{tr} A) \lambda^{2}+s(A) \lambda-\operatorname{det}(A)=r,
$$


where $r$ is either of the two roots of

$$
r^{2}+4 \Phi(a, b, c) r-|[a, \bar{b}, c]|^{2}=0 .
$$

\section{Geršgorin type theorems for matrices in $F^{n \times n}$}

Let $F$ denote any one of the spaces $R, C, \mathcal{H}$, and $\mathcal{O}$. For $A=\left[a_{i j}\right] \in F^{n \times n}$, we let

$$
R_{i}(A):=\sum_{j=1, j \neq i}^{n}\left|a_{i j}\right| .
$$

The following two results are routine generalizations of classical Geršgorin theorem and the Geršgorin type theorems of Zhang [15]. We state them for completeness.

Theorem 7 (Geršgorin type theorem for left eigenvalues). For $A=\left[a_{i j}\right] \in F^{n \times n}$, we have

$$
\sigma_{l}(A) \subseteq \bigcup_{i=1}^{n}\left\{\lambda \in F:\left|\lambda-a_{i i}\right| \leqslant R_{i}(A)\right\} .
$$

In what follows, we say that elements $\mu$ and $\lambda$ in $F$ are similar (and write $\mu \sim \lambda$ ) if there is a nonzero $z \in F$ such that $\mu=z \lambda z^{-1}$. (Note that $z \lambda z^{-1}$ is well defined even in $\mathcal{O}$ because of the alternative property that the (sub)algebra generated by any two elements in $\mathcal{O}$ is associative.)

Theorem 8 (Geršgorin theorem for right eigenvalues). Let $A=\left[a_{i j}\right] \in F^{n \times n}$. Then for every right eigenvalue $\lambda$ of $A$ there exists $\mu \in F, \mu \sim \lambda$ such that

$$
\mu \in \bigcup_{i=1}^{n}\left\{\gamma \in F:\left|\gamma-a_{i i}\right| \leqslant R_{i}(A)\right\} .
$$

\section{Strict diagonal dominance in $F^{n \times n}$}

Let $F$ be as in the previous section. For a matrix $A=\left[a_{i j}\right] \in F^{n \times n}$, we say that $A$ is strictly diagonally dominant if

$$
\left|a_{i i}\right|>R_{i}(A) \quad \forall i=1,2, \ldots, n .
$$

Theorem 9. For $A=\left[a_{i j}\right] \in F^{n \times n}$, consider the following statements:

(1) A is strictly diagonally dominant.

(2) The implication $\left[x \in F^{n}, A x=0\right] \Rightarrow x=0$ holds.

(3) There exist unique matrices $B$ and $C$ in $F^{n \times n}$ (which are equal when $A$ is defined over $F \subseteq \mathcal{H}$ ) such that $A B=I=C A$.

(4) $A$ is invertible in the Euclidean Jordan algebra $\operatorname{Herm}\left(\mathcal{H}^{n \times n}\right)$.

(5) $A$ is invertible in the Euclidean Jordan algebra Herm $\left(\mathcal{O}^{3 \times 3}\right)$.

Then we have the following implications:
(1) $\Rightarrow(2) \Leftrightarrow(3)$,
(3) $\Leftrightarrow$ (4) when $A \in \operatorname{Herm}\left(\mathcal{H}^{n \times n}\right)$

and

$$
\text { (1) } \Rightarrow \text { (5) when } A \in \operatorname{Herm}\left(\mathcal{O}^{3 \times 3}\right) \text {. }
$$


Proof. The implication (1) $\Rightarrow$ (2) follows immediately from Theorem 7.

The equivalence of (2) and (3) is obvious when $F$ is $R$ or $C$, follows from Theorem 1 when $F$ is $\mathcal{H}$ or

Now assume that $A$ belongs to $\operatorname{Herm}\left(\mathcal{H}^{n \times n}\right)$.

(3) $\Rightarrow(4)$ : When (3) holds, there exists a unique matrix $B \in \mathcal{H}^{n \times n}$ such that $A B=B A=I$. By uniqueness of $B$, we see that $B^{*}=B$, which means that $B$ is Hermitian. To prove that $B$ is the inverse of $A$ in the algebra $\operatorname{Herm}\left(\mathcal{H}^{n \times n}\right)$, we need only to show that $A$ and $B$ operator commute, that is,

$$
L_{A} L_{B}=L_{B} L_{A},
$$

where $L_{A}(X):=\frac{A X+X A}{2}$ and $L_{B}(X):=\frac{B X+X B}{2}$ for all $X \in \operatorname{Herm}\left(\mathcal{H}^{n \times n}\right)$. This easily follows due to the associativity in $\mathcal{H}$.

(4) $\Rightarrow(3)$ : As $A \in \operatorname{Herm}\left(\mathcal{H}^{n \times n}\right)$, by Theorem $1, A$ can be expanded as

$$
A=\sum_{m=1}^{n} \lambda_{m} v_{m} v_{m}^{*}
$$

where $\left\{v_{m}: m=1, \ldots, n\right\}$ is an orthonormal basis of eigenvectors of $A$, with real eigenvalues $\lambda_{m}$. In view of the properties of $v_{m}$, the set

$\left\{v_{1} v_{1}^{*}, \ldots, v_{n} v_{n}^{*}\right\}$ is a Jordan frame in $\operatorname{Herm}\left(\mathcal{H}^{n \times n}\right)$. This means that $(8)$ is the spectral decomposition of $A$ in $\operatorname{Herm}\left(\mathcal{H}^{n \times n}\right)$. Now suppose condition (4) holds. Then each $\lambda_{m}$ is nonzero. Now define

$$
B:=\sum_{m=1}^{n} \frac{1}{\lambda_{m}} v_{m} v_{m}^{*} \text {. }
$$

Then, due to properties of $v_{m}$ and associativity in $\mathcal{H}$, we have $A B=B A=I$. Moreover, due to the associativity in $\mathcal{H}$, for $C, D \in \mathcal{H}^{n \times n}, A C=D A=I \Rightarrow C=B=D$. Hence (3) holds.

We remark that it is possible to prove the implication (4) $\Rightarrow$ (3) without using (8). For example, we can show that $A B=B A=I$ when $B$ is the inverse of $A$ in $\operatorname{Herm}\left(\mathcal{H}^{n \times n}\right)$, i.e, when $B$ operator commutes with $A$ and $A \circ B=I$.

Finally, assume that $A \in \operatorname{Herm}\left(\mathcal{O}^{3 \times 3}\right)$.

$(1) \Rightarrow(5)$ : Let $A$ be strictly diagonally dominant. As $\mathcal{O}$ is non-associative, the argument of $(3) \Rightarrow$ (4) cannot be used here. So, we offer a different proof. Let

$$
A=\left[\begin{array}{lll}
p & a & b \\
\bar{a} & q & c \\
\bar{b} & \bar{c} & r
\end{array}\right],
$$

where $p, q, r \in R$ and $a, b, c \in \mathcal{O}$. Next, suppose that $A$ is not invertible in $\operatorname{Herm}\left(\mathcal{O}^{3 \times 3}\right)$ which means that one of the spectral eigenvalues of $A$ is zero, that is, $\operatorname{det}(A)=0$. Thus, from (7),

$$
0=\operatorname{det} A=p q r+2 \operatorname{Re}(\bar{b}(a c))-r|a|^{2}-q|b|^{2}-p|c|^{2} .
$$

This implies that

$$
|p q r|=\left.|-2 \operatorname{Re}(\bar{b}(a c))+r| a\right|^{2}+q|b|^{2}+p|c|^{2}|\leqslant 2| a\|b\| c|+| r\left\|\left.a\right|^{2}+\left|q \left\|\left.b\right|^{2}+|p \| c|^{2},\right.\right.\right.
$$

hence

$$
|p\|q\| r|-2|a\|b\| c|-\left(\left|r \left\|\left.a\right|^{2}+\left|q\left\|\left.b\right|^{2}+|p \| c|^{2} \mid\right) \leqslant 0 .\right.\right.\right.\right.
$$

Now, as $A$ is strictly diagonally dominant, the matrix

$$
B:=\left[\begin{array}{ccc}
|p| & -|a| & |b| \\
-|a| & |q| & |c| \\
|b| & |c| & |r|
\end{array}\right]
$$

is a real symmetric strictly diagonally dominant matrix with a positive diagonal. By a well-known matrix theory result (see [10, Theorem 6.1.10]) $B$ is positive definite and hence

$\operatorname{det} B>0$. 
Therefore,

$$
|p||q||r|-2|a||b||c|-\left(\left|r \left\|\left.a\right|^{2}+\left|q\left\|\left.b\right|^{2}+|p \| c|^{2} \mid\right)>0,\right.\right.\right.\right.
$$

which is clearly a contradiction. Hence $A$ is invertible in $\operatorname{Herm}\left(\mathcal{O}^{3 \times 3}\right)$.

Remark 2. The following example shows that the implication $(2) \Rightarrow(5)$ fails for octonion matrices. In $\operatorname{Herm}\left(\mathcal{O}^{3 \times 3}\right)$, let

$$
A=\left[\begin{array}{ccc}
\sqrt{3} & e_{2} & e_{6} \\
-e_{2} & \sqrt{3} & e_{1} \\
-e_{6} & -e_{1} & \sqrt{3}
\end{array}\right] .
$$

Then, using (7) and the multiplication table for $\mathcal{O}, \operatorname{det}(A)=0$, and so zero is a spectral eigenvalue of $A$. This means that $A$ is not invertible in the algebra $\operatorname{Herm}\left(\mathcal{O}^{3 \times 3}\right)$. We claim that zero is not a left/right eigenvalue of $A$. Assuming the contrary, by Lemma $6, \lambda=0$ must satisfy

$$
\operatorname{det}(\lambda I-A)=\lambda^{3}-(\operatorname{tr}(A)) \lambda^{2}+s(A) \lambda-\operatorname{det} A=r,
$$

where $r$ is either of the two roots of

$$
r^{2}+4 \Phi\left(e_{2}, e_{6}, e_{1}\right) r-\left|\left[e_{2},-e_{6}, e_{1}\right]\right|^{2}=0
$$

with $s(A)$ and $\Phi$ previously defined. Thus, $0=-\operatorname{det}(A)=r$. Now,

$$
\left|\left[e_{2},-e_{6}, e_{1}\right]\right|^{2}=\left|-2 e_{5}\right|^{2}=4 \neq 0 ;
$$

hence $r \neq 0$, leading to a contradiction.

Thus, zero is not a real eigenvalue of $A$, even though, it is a spectral eigenvalue of $A$. In particular, we have $A x=0 \Rightarrow x=0$.

Remark 3. In the context of $\operatorname{Herm}\left(R^{n \times n}\right)$ or $\operatorname{Herm}\left(C^{n \times n}\right)$, it is well known that if $X$ and $Y$ are positive semidefinite matrices (that is, they belong to the symmetric cone), then

$$
X \circ Y=0 \Leftrightarrow\langle X, Y\rangle=0 \Leftrightarrow X Y=0 .
$$

In this remark, we will demonstrate that these equivalences continue to hold in $\operatorname{Herm}\left(\mathcal{H}^{n \times n}\right)$, but that the second equivalence fails in $\operatorname{Herm}\left(\mathcal{O}^{3 \times 3}\right)$.

It is known that in any Euclidean Jordan algebra $V$ with corresponding symmetric cone $K$, the following two statements are equivalent, see [8, Proposition 6]:

(i) $x \in K, y \in K$, and $x \circ y=0$.

(ii) $x \in K, y \in K$, and $\langle x, y\rangle=0$.

Moreover, in each case, the objects $x$ and $y$ operator commute. Thus, to see (9) in $\operatorname{Herm}\left(\mathcal{H}^{n \times n}\right.$ ) (or for that matter, in $\operatorname{Herm}\left(R^{n \times n}\right)$ or $\left.\operatorname{Herm}\left(C^{n \times n}\right)\right)$, it is enough to show that

$X$ and $Y$ positive semidefinite in $\operatorname{Herm}\left(\mathcal{H}^{n \times n}\right),\langle X, Y\rangle=0 \Rightarrow X Y=0$.

In view of the operator commutativity and the spectral decomposition theorem, this reduces to showing:

If $F_{1}$ and $F_{2}$ are two primitive idempotents in $\operatorname{Herm}\left(\mathcal{H}^{n \times n}\right)$ with $\operatorname{Re} \operatorname{tr}\left(F_{1} F_{2}\right)=0$, then $F_{1} F_{2}=0$.

Now if $F_{1}$ and $F_{2}$ are two primitive idempotents in $\operatorname{Herm}\left(\mathcal{H}^{n \times n}\right)$, then as in (8) we can expand $F_{1}$ and $F_{2}$ using their eigenvalues and eigenvectors:

$$
F_{1}=v v^{*} \text { and } F_{2}=w w^{*}
$$

where $v$ and $w$ are unit quaternion vectors. If $\operatorname{Re} \operatorname{tr}\left(F_{1} F_{2}\right)=0$, then $\operatorname{Re} \operatorname{tr}\left(v v^{*} w w^{*}\right)=0$. Putting $c:=$ $v^{*} w$, expanding $\operatorname{Re} \operatorname{tr}\left(v v^{*} w w^{*}\right)$ as a sum and using the fact that $\operatorname{Re}(a b-b a)=0$ for any two quaternions, we see that $\operatorname{Re} \operatorname{tr}\left(v v^{*} w w^{*}\right)=\operatorname{Re}(c \bar{c})$. Thus, $0=\operatorname{Re}(c \bar{c})$ and so $v^{*} w=c=0$. From this, we get 
$F_{1} F_{2}=v v^{*} w w^{*}=0$. Thus we have (9) for quaternion Hermitian matrices. Now we claim that the second equivalence in (9) fails for octonions.

Consider the matrix $A$ given in the previous example. We write the spectral decomposition for this A:

$$
A=0 F_{1}+\lambda_{2} F_{2}+\lambda_{3} F_{3},
$$

where $\left\{F_{1}, F_{2}, F_{3}\right\}$ is a Jordan frame in $\operatorname{Herm}\left(\mathcal{O}^{3 \times 3}\right)$ and $\sigma_{s p}(A)=\left\{0, \lambda_{2}, \lambda_{3}\right\}$. We claim that both $F_{2} F_{1}$ and $F_{3} F_{1}$ cannot be zero simultaneously. Assuming the contrary, we have $F_{2} F_{1}=0$ and $F_{3} F_{1}=0$; hence

$$
A F_{1}=0 .
$$

Now if $u$ is any column of $F_{1}$, then $A u=0$. By the known property of $A$ (see the end of previous remark), we must have $u=0$ proving $F_{1}=0$. But this is a contradiction as $F_{1}$ is a primitive idempotent and hence cannot be zero.

Remark 4. The following example shows that the implication (5) $\Rightarrow(2)$ in Theorem 9 need not be true.

Let

$$
\begin{aligned}
& A:=\left[\begin{array}{ccc}
1 & e_{2} & e_{6} \\
-e_{2} & 1 & e_{1} \\
-e_{6} & -e_{1} & 1
\end{array}\right], \\
& x_{1}:=1+e_{1}+e_{2}+e_{3}+e_{4}-e_{5}-e_{6}-e_{7}, \quad x_{2}:=0, \quad \text { and } \\
& x_{3}:=1+e_{1}-e_{2}-e_{3}+e_{4}-e_{5}+e_{6}+e_{7} .
\end{aligned}
$$

Then

$$
A\left[\begin{array}{l}
x_{1} \\
x_{2} \\
x_{3}
\end{array}\right]=0
$$

hence 0 is a left/right eigenvalue of $A$. By the modified characteristic equation in Lemma 6 , we get $-\operatorname{det}(A)=r$. Solving for $r$ from

$$
r^{2}+4 \Phi\left(e_{2}, e_{6}, e_{1}\right) r-\left|\left[e_{2},-e_{6}, e_{1}\right]\right|^{2}=0,
$$

we get $r= \pm 2$ and so $\operatorname{det}(A) \neq 0$. Hence 0 is a not a spectral eigenvalue of $A$.

Examples in Remarks 2 and 4 show that for matrices in $\operatorname{Herm}\left(\mathcal{O}^{3 \times 3}\right)$, the spectral eigenvalues and real left/right eigenvalues of $A$ can be different.

\section{Strict diagonal dominance in Euclidean Jordan algebras}

Theorem 10. Let $(V, \circ,\langle\cdot, \cdot\rangle)$ be any Euclidean Jordan algebra of rank $r$ and

$$
x=\sum_{i=1}^{r} x_{i} e_{i}+\sum_{i<j} x_{i j}
$$

be the Peirce decomposition of $x \in V$ with respect to a given Jordan frame $\left\{e_{1}, \ldots, e_{r}\right\}$. If $x$ is strictly diagonally dominant, that is, if

$$
\left|x_{i}\right|>R_{i}(x):=\frac{1}{\sqrt{2}\left\|e_{i}\right\|}\left(\sum_{k=1}^{i-1}\left\|x_{k i}\right\|+\sum_{j=i+1}^{r}\left\|x_{i j}\right\|\right) \quad \forall i=1,2, \ldots, r,
$$

then $x$ is invertible in $V$. 
Proof. We first suppose that $V$ is simple.

Case 1: Let $V$ be one of the matrix algebras. We note that if the Peirce decomposition of $x$ is strictly diagonally dominant with respect to the Jordan frame $\left\{e_{1}, e_{2}, \ldots, e_{r}\right\}$, then for any algebra automorphism $\Lambda$ on $V$, the Peirce decomposition of $\Lambda(x)$ is strictly diagonally dominant with respect to $\left\{\Lambda\left(e_{1}\right), \Lambda\left(e_{2}\right), \ldots, \Lambda\left(e_{r}\right)\right\}$ (as any algebra automorphism on a simple algebra is orthogonal, see Section 3). As $V$ is simple, any Jordan frame can be mapped onto another (see Section 3). Hence we assume, without loss of generality, that the Jordan frame is the canonical one given by $\left\{E_{1}, E_{2}, \ldots, E_{r}\right\}$ where $E_{i}$ is the matrix with one in the $(i, i)$ slot and zeros elsewhere. Now if $x$ is strictly diagonally dominant with respect to this Jordan frame, we can apply Theorem 9 and get the invertibility.

Case 2: Now assume that $V=\mathcal{L}^{n}$. Let $x=x_{1} e_{1}+x_{2} e_{2}+x_{12}$ be the Peirce decomposition of $x$ with respect to a Jordan frame $\left\{e_{1}, e_{2}\right\}$. Given

$$
\left|x_{1}\right|>R_{1}(x), \quad\left|x_{2}\right|>R_{2}(x),
$$

we have to show that $x$ is invertible in $\mathcal{L}^{n}$. Now (6) shows that $R_{1}(x)=\left\|x_{12}\right\|=R_{2}(x)$. Also, from (5),

$$
\operatorname{det}(x)=x_{1} x_{2}-\left\|x_{12}\right\|^{2} .
$$

We see that $\operatorname{det}(x) \neq 0$ proving the invertibility of $x$. Thus, we have proved the invertibility of $x$ when $V$ is one of the standard simple algebras.

Note that the result continues to hold in each of these standard algebras when we change the inner product to a constant multiple of the trace inner product. (The reason being that the Peirce decomposition remains the same except that the norms of objects get multiplied by a constant factor.) Now, using the structure theorem (see Section 3 ) and the fact that in any simple algebra, the inner product is a multiple of the trace product (see Prop. III.4.1 in [6]), we can prove our result in any simple Euclidean Jordan algebra.

Now let $V$ be any Euclidean Jordan algebra. By the structure theorem, we can write $V=V_{1} \times V_{2} \times$ $\cdots \times V_{k}$ where each $V_{i}$ is simple. For notational simplicity, we let $k=2$ and put $r_{1}=\operatorname{rank}\left(V_{1}\right), r_{2}=$ $\operatorname{rank}\left(V_{2}\right)$. We regard any element of $V$ as a column vector with two components, the first component belonging to $V_{1}$ and the second component belonging to $V_{2}$. If $c$ is any primitive idempotent in $V$, then exactly one component of $c$ is nonzero and this nonzero component is a primitive idempotent in the corresponding component algebra. By rearranging the elements, we may write

$$
\left\{e_{1}, e_{2}, \ldots, e_{r}\right\}=\left\{\left[\begin{array}{c}
g_{1} \\
0
\end{array}\right],\left[\begin{array}{c}
g_{2} \\
0
\end{array}\right], \ldots,\left[\begin{array}{c}
g_{r_{1}} \\
0
\end{array}\right],\left[\begin{array}{c}
0 \\
h_{1}
\end{array}\right], \ldots,\left[\begin{array}{c}
0 \\
h_{r_{2}}
\end{array}\right]\right\}
$$

where $\left\{g_{1}, g_{2}, \ldots, g_{r_{1}}\right\}$ is a Jordan frame in $V_{1}$ and $\left\{h_{1}, h_{2}, \ldots, h_{r_{2}}\right\}$ is a Jordan frame in $V_{2}$. Now writing the given element $x$ as a column vector with two components $u \in V_{1}$ and $v \in V_{2}$, we may write the Peirce decomposition of $x$ in the form

$$
x=\sum_{i=1}^{r_{1}} u_{i}\left[\begin{array}{c}
g_{i} \\
0
\end{array}\right]+\sum_{i<j \leqslant r_{1}}\left[\begin{array}{c}
u_{i j} \\
0
\end{array}\right]+\sum_{i=1}^{r_{2}} v_{i}\left[\begin{array}{c}
0 \\
h_{i}
\end{array}\right]+\sum_{i<j \leqslant r_{2}}\left[\begin{array}{c}
0 \\
v_{i j}
\end{array}\right],
$$

where we have used the fact that the Peirce space $V_{i j}$ with respect to any pair $\left\{\left[\begin{array}{c}g_{i} \\ 0\end{array}\right],\left[\begin{array}{c}0 \\ h_{j}\end{array}\right]\right\}$ is zero. The strict diagonal dominance of $x$ now implies that $u$ and $v$ are strictly diagonally dominant with respect to $\left\{g_{1}, g_{2}, \ldots, g_{r_{1}}\right\}$ in $V_{1}$ and $\left\{h_{1}, h_{2}, \ldots, h_{r_{2}}\right\}$ in $V_{2}$. By our previous arguments, $u$ and $v$ are invertible in $V_{1}$ and $V_{2}$ respectively. It follows that $x$ is invertible in $V$. This concludes the proof of the theorem.

\section{A Geršgorin type theorem in Euclidean Jordan algebras}

Theorem 11. Let $V$ be a Euclidean Jordan algebra of rank $r$ and

$$
x=\sum_{i=1}^{r} x_{i} e_{i}+\sum_{i<j} x_{i j}
$$

be the Peirce decomposition of $x \in V$ with respect to a given Jordan frame $\left\{e_{1}, \ldots, e_{r}\right\}$. Then 


$$
\sigma_{s p}(x) \subseteq \bigcup_{i=1}^{r}\left\{\lambda \in R:\left|\lambda-x_{i}\right| \leqslant R_{i}(x)\right\},
$$

where $R_{i}(x)$ is given by (2). Moreover, if a union of $k$ Geršgorin intervals forms an interval that is disjoint from the remaining $n-k$ Geršgorin intervals, then there are precisely $k$ spectral eigenvalues of $x$ in this interval.

Note. It is possible to say precisely which $k$ spectral eigenvalues lie in the union of $k$ Geršgorin intervals, see the proof below.

Proof. Suppose that the stated inclusion fails, so that there exists a $\lambda \in \sigma_{s p}(x)$ such that $\left|\lambda-x_{i}\right|>R_{i}(x)$, for all $i=1, \ldots, r$. Then $y:=x-\lambda e$ has the Peirce decomposition

$$
y=\sum_{i=1}^{r}\left(x_{i}-\lambda\right) e_{i}+\sum_{i<j} x_{i j}
$$

and hence is a strictly diagonally dominant element of $V$. By Theorem 10, $y$ is invertible. Now let

$$
x=\lambda_{1} f_{1}+\cdots+\lambda_{r} f_{r}
$$

be the spectral decomposition of $x$, where $\left\{f_{1}, \ldots, f_{r}\right\}$ is a Jordan frame.

Then

$$
y=\left(\lambda_{1}-\lambda\right) f_{1}+\cdots+\left(\lambda_{r}-\lambda\right) f_{r}
$$

is the spectral decomposition of $y$. As $\lambda \in \sigma_{s p}(x)=\left\{\lambda_{1}, \lambda_{2}, \ldots, \lambda_{r}\right\}, \lambda_{i}=\lambda$, for some $i$. It follows that zero is a spectral eigenvalue of $y$ which means that $y$ is not invertible. This is a contradiction. Hence we have the spectral inclusion.

Now for the second part of the theorem. Its proof, as in the classical case of complex matrices (see [10, p. 345]), relies on continuity of eigenvalues.

First suppose that $V$ is simple. Define

$$
x(\varepsilon):=\sum_{i=1}^{r} x_{i} e_{i}+\varepsilon \sum_{i<j} x_{i j}
$$

with $\varepsilon \in[0,1]$. Note that $x(1)=x$ and $x(0)=\sum_{i=1}^{r} x_{i} e_{i}$. Also,

$$
R_{i}(x(\varepsilon)) \leqslant R_{i}(x)
$$

for each $i$ and so the spectrum of $x(\varepsilon)$ is contained in the union of Geršgorin intervals of $x$.

Now we consider the decreasing rearrangement of spectral eigenvalues of $x(\varepsilon)$ :

$$
\lambda^{\downarrow}(x(\varepsilon)):=\left[\begin{array}{llll}
\lambda_{1}^{\downarrow}(x(\varepsilon)) & \lambda_{2}^{\downarrow}(x(\varepsilon)) & \cdots & \lambda_{r}^{\downarrow}(x(\varepsilon))
\end{array}\right]^{T},
$$

where $\lambda_{1}^{\downarrow}(x(\varepsilon)) \geqslant \lambda_{2}^{\downarrow}(x(\varepsilon)) \geqslant \cdots \geqslant \lambda_{r}^{\downarrow}(x(\varepsilon))$. In particular, for $\varepsilon=0$,

$$
\lambda^{\downarrow}(x(0))=\left[\begin{array}{llll}
x_{1}^{\downarrow} & x_{2}^{\downarrow} & \cdots & x_{r}^{\downarrow}
\end{array}\right]^{T} .
$$

In view of the continuity of $\lambda^{\downarrow}(x(\varepsilon))$ in $\varepsilon$ (see e.g., Theorem 9 in [9]) each of the spectral eigenvalue curves joining $x_{i}^{\downarrow}$ and $\lambda_{i}^{\downarrow}(x)$ lies in the union of all Geršgorin intervals of $x$. Now consider the union of $k$ Geršgorin intervals that form an interval (i.e., a connected set) which is disjoint from other Geršgorin intervals of $x$. Corresponding to the center, say, $x_{i}^{\downarrow}$ of a Geršgorin interval that is contained in this union, the other end of the spectral eigenvalue curve, namely, $\lambda_{i}^{\downarrow}(x)$ must also be in this union. Even the converse statement holds. Thus there are exactly $k$ eigenvalues of $x$ that lie in this union.

Now let $V$ be a general Euclidean Jordan algebra and let $k$ Geršgorin intervals of $x$ form an interval that is disjoint from other Geršgorin intervals of $x$. Define $x(\varepsilon)$ as in the previous case. Suppose, without 
loss of generality, $x_{1}$ is the center of one of the Geršgorin intervals in this union. Then the associated primitive idempotent $e_{1}$ (in the Peirce decomposition of $x$ with respect to $\left\{e_{1}, e_{2}, \ldots, e_{r}\right\}$ ) belongs to a unique factor (simple) algebra, say, $V_{1}$ of $V$. Using the continuity of spectral eigenvalues in simple algebras (as observed above), we can conclude that the spectral eigenvalue curve joining $x_{1}$ and one of the spectral eigenvalues of $x$ lies in this union. Conversely, each spectral eigenvalue of $x$ that lies in this union connects to one of the centers that lies in the union. Because of this one-to-one correspondence, we see that there are exactly $k$ spectral eigenvalues of $x$ lying in the union. This completes the proof.

It is well known that an object $x$ of $V$ belongs to $K$ (interior of $K$ ) if and only if all the spectral eigenvalues of $x$ are nonnegative (respectively, positive). The following result is an immediate consequence of the above theorem.

Corollary 12. If in the above theorem, $x$ is strictly diagonally dominant with respect to some Jordan frame and the diagonal elements $x_{i}$ are positive, then $x$ is in the interior of the symmetric cone.

\section{References}

[1] J.C. Baez, The octonions, Bull. Amer. Math. Soc., 39 (2002) 145-205

[2] T. Dray, J. Janesky, C.A. Manogue, Octonionic Hermitian matrices with non-real eigenvalues, Adv. Appl. Clifford Alg. 10 (2000) 193-216

[3] T. Dray, C.A. Manogue, The octonionic eigenvalue problem, Adv. Appl. Clifford Alg. 8 (1998) 341-364.

[4] T. Dray, C.A. Manogue, The exceptional Jordan eigenvalue problem, Internat. J. Theoret. Phys. 38 (1999) 2901-2916.

[5] T. Dray, C.A. Manogue, S. Okubo, Orthogonal eigenbases over the octonions, Algebras Groups Geom. 19 (2002) 163-180.

[6] J. Faraut, A. Korányi, Analysis on Symmetric Cones, Clarendon Press, Oxford, 1994.

[7] H. Freudenthal, Lie groups in the foundations of geometry, Adv. Math. 1 (1964) 145-190.

[8] M.S. Gowda, R. Sznajder, J. Tao, Some P-properties for linear transformations on Euclidean Jordan algebras, Linear Algebra Appl. 393 (2004) 203-232

[9] M.S. Gowda, J. Tao, M. Moldovan, Some inertia theorems in Euclidean Jordan algebras, Linear Algebra Appl. 430 (2009) 1992-2011.

[10] R.A. Horn, C.R. Johnson, Matrix Analysis, Cambridge University Press, Cambridge, 1985.

[11] S.H. Schmieta, F. Alizadeh, Extension of primal-dual interior point algorithms to symmetric cones, Math. Prog. Ser. A 96 (2003) 409-438.

[12] J. Tao Some P-Properties for Linear Transformations on the Lorentz Cone, Ph.D. Thesis, UMBC, 2004

13] Y. Tian, Matrix representations of octonions and their applications, Adv. Appl. Clifford Alg. 10 (2000) 61-90

[14] F. Zhang, Quaternions and matrices of quaternions, Linear Algebra Appl. 251 (1997) 21-57.

[15] F. Zhang, Geršgorin type theorem for quaternionic matrices, Linear Algebra Appl. 424 (2007) 139-153. 